\title{
Automated Segmentation of 3-D Spectral OCT Retinal Blood Vessels by Neural Canal Opening False Positive Suppression
}

\author{
Zhihong $\mathrm{Hu}^{1}$, Meindert Niemeijer ${ }^{1,2}$, Michael D. Abràmoff' ${ }^{2,1,3}$, \\ Kyungmoo Lee ${ }^{1}$, and Mona K. Garvin ${ }^{1}$ \\ ${ }^{1}$ Departments of Electrical and Computer Engineering, and \\ ${ }^{2}$ Ophthalmology and Visual Sciences, The University of Iowa, Iowa City, IA, USA \\ ${ }^{3}$ Iowa City VA Medical Center, Iowa City, IA, USA \\ \{zhihong-hu,mona-garvin\}@uiowa.edu
}

\begin{abstract}
We present a method for automatically segmenting the blood vessels in optic nerve head $(\mathrm{ONH})$ centered spectral-domain optical coherence tomography (SD-OCT) volumes, with a focus on the ability to segment the vessels in the region near the neural canal opening (NCO). The algorithm first pre-segments the NCO using a graph-theoretic approach. Oriented Gabor wavelets rotated around the center of the NCO are applied to extract features in a $2-\mathrm{D}$ vessel-aimed projection image. Corresponding oriented NCO-based templates are utilized to help suppress the false positive tendency near the NCO boundary. The vessels are identified in a vessel-aimed projection image using a pixel classification algorithm. Based on the $2-\mathrm{D}$ vessel profiles, 3 -D vessel segmentation is performed by a triangular-mesh-based graph search approach in the $\mathrm{SD}-\mathrm{OCT}$ volume. The segmentation method is trained on 5 and is tested on 10 randomly chosen independent ONH-centered SD-OCT volumes from 15 subjects with glaucoma. Using ROC analysis, for the 2-D vessel segmentation, we demonstrate an improvement over the closest previous work with an area under the curve (AUC) of 0.81 ( 0.72 for previously reported approach) for the region around the $\mathrm{NCO}$ and 0.84 for the region outside the NCO (0.81 for previously reported approach).
\end{abstract}

\section{Introduction}

Spectral-domain optical coherence tomography (SD-OCT) is a noncontact, noninvasive imaging technique used to obtain high resolution images of the retina or optic nerve head (ONH). It is a powerful modality to qualitatively assess retinal features and pathologies or to make quantitative measurements of retinal or ONH morphology.

Due to the fact that the retinal blood vessels absorb the wavelengths of light used in SD-OCT, the vessels in these volumes are mostly not directly visible. However, this causes vessel shadows to appear below the position of the vessels. As reported by Wehbe et al. 11, the retinal vessels are located right above the vessel shadows in the $z$-direction of the OCT volume and these shadows can be

T. Jiang et al. (Eds.): MICCAI 2010, Part III, LNCS 6363, pp. 33-40, 2010.
(C) Springer-Verlag Berlin Heidelberg 2010 
used to detect the location of the vessels. The segmentation of vessels in SDOCT could lead to a more objective diagnosis of diseases, be used for OCT-toOCT or OCT-to-fundus registration, help remove the influence of vessels (when desirable), and aid in the detection of other retinal anatomic structures.

However, vessel segmentation in SD-OCT volumes of the $\mathrm{ONH}$ is not a trivial problem for a number of reasons. 1) The vessel shadows have a weak visibility in OCT due to the 3-D structure of the ONH. 2) Many vessels can overlap in regions, especially the region inside the neural canal opening ( $\mathrm{NCO})$, where the individual vessels cannot be discerned. 3) The presence of the NCO boundary, which crosses with the vessels, causes false positives for the vessel segmentation. 4) The vessel shadows have a decreased contrast with the background within the $\mathrm{NCO}$, due to more variable tissue properties in the $\mathrm{ONH}$.

Niemeijer et al. 22 previously presented an automated vessel segmentation algorithm for SD-OCT volumes of the ONH. In their algorithm, they first created a $2-\mathrm{D}$ projection image from the $3-\mathrm{D}$ volume and then utilized a pixel classification approach to segment the vessels in the projection image. Features were computed using Gaussian filter banks. However, their approach mainly segmented the vessels in the region outside the NCO and the vessels inside the NCO were excluded from the analysis. In addition, the algorithm exhibited a large numbers of false positives near the NCO. For example, the top row of Fig. 1 shows the NCO boundary (blue arrows) and typical false positives (red arrow) near the NCO from Niemeijer's vessel segmentation approach.

In this work, we present a 2-D pixel classification algorithm to segment the blood vessels in SD-OCT volumes centered at the ONH, with a special focus on better identifying vessels near the NCO (Section 2.2). We approach this by incorporating pre-segmented NCO location (Section 2.1) information into the classification process. We then utilize these 2-D vessel profiles to segment the $3-\mathrm{D}$ vessels in the SD-OCT volumes (Section 2.2).

\section{Methods}

\subsection{Neural Canal Opening Pre-segmentation}

In order to incorporate the NCO information into the 2-D vessel classification process, a graph-theoretic approach is first applied to identify the location of the NCO [3. More specifically, the ONH-centered raw OCT volume is first flattened by identifying four intraretinal surfaces using a graph-search-based multilayer segmentation algorithm in 3-D, fitting a thin-plate spline to a segmented surface by ignoring the central $\mathrm{ONH}$ region, and then translating the columns so that this surface becomes a flat plane [4. For surface 2, 3, and 4, the average positions outside an estimated $\mathrm{NCO}$ region are radially interpolated into the inside region. A 2-D projection image is obtained by computing the mean intensity values from a small number of slices between the interpolated surface 2 (orange) and 4 (yellow) (Fig.13). Thus the 3-D NCO segmentation problem is converted to 


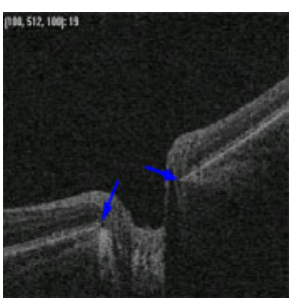

(a)

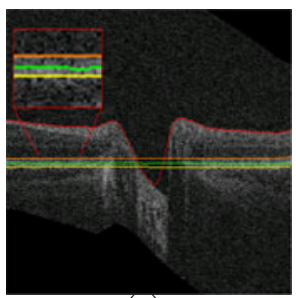

(e)

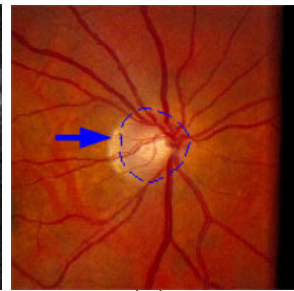

(b)

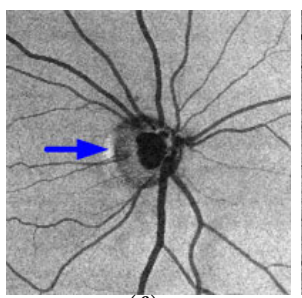

(f)

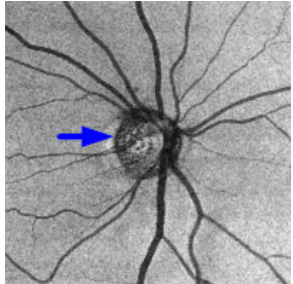

(c)

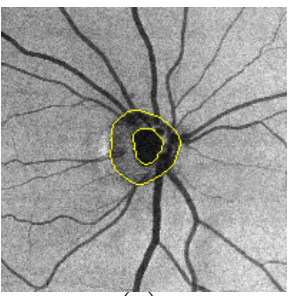

(g)

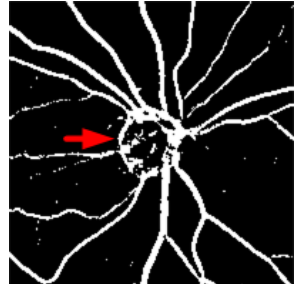

(d)

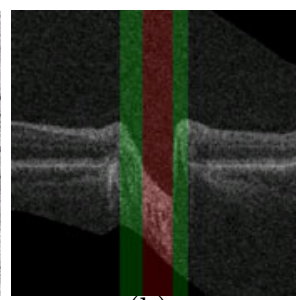

(h)

Fig. 1. Niemeijer's vessel segmentation approach and our neural canal opening segmentation. (Top row) An example illustration of the NCO boundary (indicated with blue arrow) and false positives of NCO (indicated with red arrow) by Niemeijer's vessel segmentation approach 2]. (a) Central slice of an OCT volume. (b) Corresponding fundus image. Note that the blue-arrow indicated contour is the NCO boundary. (c) OCT projection image. (d) Vessel segmentation result showing the typical false positives near the NCO. (Bottom row) An example illustration of our NCO segmentation algorithm [3]. (e) 3-D four surface segmentation with the interpolation of the radial average positions outside the estimated NCO to inside the NCO for surface 2 (orange), 3 (green), and 4 (yellow). (f) NCO-aimed projection image from the layer between the interpolated surface 2 and 4 . Segmented NCO and cup overlapping with (g) the projection image and (h) a cross-sectional slice of the OCT volume.

a 2-D problem. The projection image is referred as the "NCO-aimed projection image" (Fig. 1f). The outer boundary in the projection image corresponds to the $\mathrm{NCO}$ and the inner boundary corresponds to the cup at the level of the NCO reference plane.

In order to perform the graph search, the NCO-aimed projection image is transformed to polar coordinates by unwrapping from the geometric center of the image. A signed edge-based term favoring a dark-to-bright transition from the transformed projection image is used as the cost function. In our case, the unwrapped cost image can be modeled as a weighted, directed graph similar to the one described by $\mathrm{Li}$ et al. [5] but reduced to a two-dimensional problem. We use the graph search to simultaneously segment the optimal NCO and cup boundaries. The NCO and cup boundaries are finally smoothed using a B-spline. The bottom row of Fig. 1 illustrates the NCO-aimed projection image creation and NCO pre-segmentation. 


\subsection{Blood Vessel Segmentation}

For the 2-D vessel segmentation, oriented Gabor wavelets around the center of the $\mathrm{NCO}$ are utilized to extract features in a $2-\mathrm{D}$ vessel-aimed projection image. The corresponding oriented NCO-based templates are utilized along with the Gabor wavelets to suppress the false positive tendency near the NCO boundary. A supervised pixel classification algorithm is applied to automatically segment the blood vessels in the vessel-aimed projection image. Based on the 2-D vessel location information, the $3-\mathrm{D}$ vessels are detected by applying a triangularmesh-based graph search to the isotropic SD-OCT volume [6]. These steps are described in more detail below.

Vessel-aimed projection image creation. The main difficulty of vessel segmentation within SD-OCT volumes is the weak visibility of the vessel pattern. In the previous approach [2, they proposed to use a 2-D projection of the vessel pattern from the 3-D volume to segment vessels. They compared two different projection images: the "naive" projection image computed by averaging the whole OCT volume which decreased the contrast between the vessels and background and the "smart" projection image computed by averaging the layer between surface 2 and 4 which provided a good contrast. However, the "smart" projection image also had some disadvantages. For example, near and inside the $\mathrm{NCO}$, the layer between surface 2 and 4 would frequently become very thin (and be subject to layer segmentation errors) and thus the projection image would not necessarily demonstrate an optimal contrast between vessels and background in this region.

In this work, we create a new type of projection image by averaging the layer between surface 2 and an under planar surface defined by the deepest position of the top surface. In columns for which the deepest position is above the interpolated surface 4, the projection image is created as that used for the "smart" projection image. The created new projection is referred as the "vessel-aimed projection image". Compared with the "naive" and "smart" projection images [2], the vessel-aimed projection image relies less on the surface segmentation and can take advantage of the vessel information inside the NCO. An illustration of the bounding surfaces for the three different projection images is shown in Fig. 2ar.

2-D vessel segmentation. In the vessel-aimed projection image, the blood vessels generally radially distribute around the NCO center. Gabor wavelets demonstrate some desirable characteristics: spatial frequency, spatial locality, and orientation selectivity. The nature of the Gabor wavelets makes them well suitable for the feature generation of the blood vessel detection. Recall that a Gabor wavelet $\psi_{\mu, \nu}(z)$ [7] can be defined as:

$$
\psi_{\mu, \nu}(z)=\frac{\left\|k_{\mu, \nu}\right\|^{2}}{\sigma^{2}} e^{-\frac{\left\|k_{\mu, \nu}\right\|^{2}\|z\| \|^{2}}{2 \sigma^{2}}}\left[e^{i k_{\mu, \nu} z}-e^{-\frac{\sigma^{2}}{2}}\right],
$$

where $z=(x, y),\|\cdot\|$ is the norm operator, $\mu$ and $\nu$ define the orientation and spatial frequency scale of the Gabor kernel, and $\sigma$ is related to the standard 


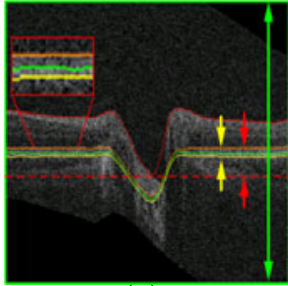

(a)

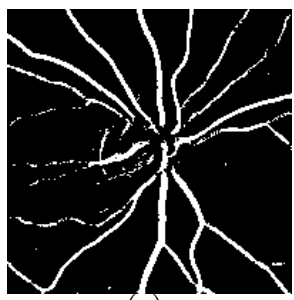

(e)

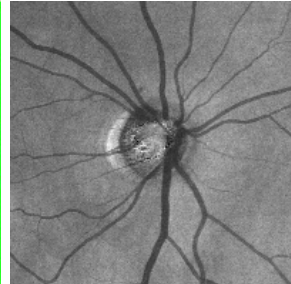

(b)

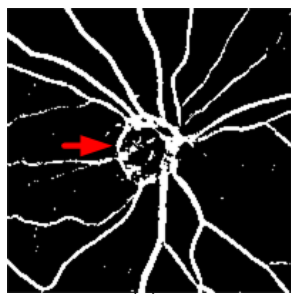

(f)

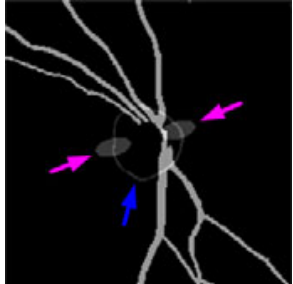

(c)

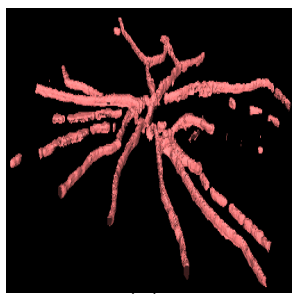

(g)

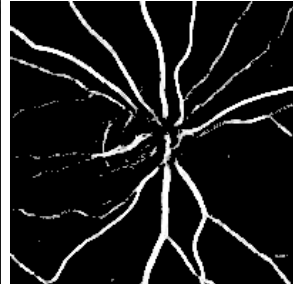

(d)

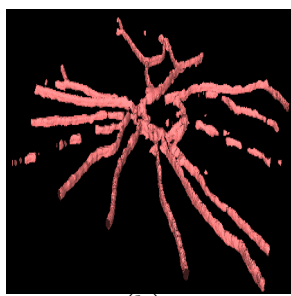

(h)

Fig. 2. Illustration of the 2-D vessel segmentation using our approach and Niemeijer's approach. (a) The bounding surfaces used for the creation of three potential projection images: "naive" (bounding surfaces of whole volume as indicated with green arrows), "smart" (orange surface 2 and yellow surface 4 as indicated by yellow arrows), and "vessel-aimed projection image" (orange surface 2 and dashed red surface where possible as indicated with red arrows; same as "smart" where the red dashed surface is above the interpolated surface 4). (b) Vessel-aimed projection image as used in this work. (c) A schmatic illustration of the Gabor wavelet responses and the NCO-based templates oriented at 165 degrees. Blue arrow $=$ NCO contour. Purple arrows = template pair centered on the NCO boundary. (d-e) 2-D vessel segmentation posterior probability and thresholded binary images of our approach. (f) 2-D vessel segmentation of Niemeijer's approach. (g-h) 3-D vessel segmentation of our approach and Niemeijer's approach.

derivation of the Gaussian window in the kernel and determines the ratio of the Gaussian window width to the wavelength. The wave vector $k_{\mu, \nu}$ is defined as

$$
k_{\mu, \nu}=k_{\nu} e^{i \phi_{\mu}}
$$

where $k_{\nu}=\frac{k_{\max }}{f^{\nu}}$ in which $k_{\max }$ is the maximum frequency and $f^{\nu}$ is the spatial frequency between kernels in the frequency domain. $\phi_{\mu}=\frac{\pi \mu}{n}$ in which $n$ is the total number of orientations. Based on the vessel profiles, in our application, we choose $k_{\max }=\frac{\pi}{2}, \sigma \in\{1,2,3\}, f=\sqrt{2}, \nu \in\{1,2,3\}, n=18$, and $\mu \in\{0, \ldots, 17\}$.

Additionally, to increase the signal and decrease the noise, three spatial frequency scale additions are applied between $\nu=2$ and $\nu=3$ in $\sigma \in\{1,2,3\}$. Together a Gabor wavelet family with 3 Gaussian scales, 3 spatial frequency scales, 3 spatial frequency scale additions, and 18 orientations is generated.

In order to suppress the false positive tendency near the NCO, the oriented templates are utilized along with the corresponding Gabor wavelets in the feature space (Fig. 2r). Specifically a pair of pre-defined templates is first created based 
on the previously segmented NCO location information and so-called NCO-based templates. The center of the template pair is that of $\mathrm{NCO}$ and the center of each of them lies on the NCO boundary. The shapes of the templates are defined as:

$$
\frac{\left\{x_{1}-x_{c}-r_{1}\right\}^{2}}{W^{2}}+\frac{\left\{y_{1}-y_{c}\right\}^{2}}{H^{2}}=1,
$$

and

$$
\frac{\left\{x_{2}-x_{c}+r_{2}\right\}^{2}}{W^{2}}+\frac{\left\{y_{2}-y_{c}\right\}^{2}}{H^{2}}=1,
$$

where $\left(x_{c}, y_{c}\right)$ is the NCO center, $r_{1}$ and $r_{2}$ are the distances of the center of each template to the center of NCO, and $W$ and $H$ are the maximum width and height of the templates which are defined based on prior knowledge of NCO profiles.

The NCO-based templates rotate in the same orientations with the Gabor wavelets. Wherever the templates rotate, the average background pixel value is assigned to those regions. The main advantage of the NCO-based templates is that it could suppress the false positive tendency from the NCO and at the same time does not affect the true positive of vessels, based on the assumption that the vessels are not parallel with the NCO.

After the feature extraction, each feature is normalized to zero mean and unit variance. A $k$-NN classifier with $k=31$ is applied for the pixel classification. Each pixel in the vessel-aimed projection test image is assigned a soft label. Together they form a posterior map. Finally a threshold filter is utilized on the posterior probability map to obtain a binary segmentation image and a voting filter is applied to remove noise and small vessels. Fig. 2 $\mathrm{d}$ and 2 e show an example result of the 2-D vessel segmentation.

3-D vessel segmentation. In order to perform the $3-\mathrm{D}$ vessel segmentation, the flattened SD-OCT is first transformed to an isotropic volume. Surface 1 and 2 are also correspondingly transformed. As mentioned above, the blood vessels themselves in the SD-OCT volume of the $\mathrm{ONH}$ are not visible. What we measure in the $2-\mathrm{D}$ vessel-aimed projection image are the vessel shadows. The "true" vessels are located right above the vessel shadows [1. We assume the vessels are approximately the three middle voxels between surface 1 and 2 in the $z$-axis of the SD-OCT volume. An initial binary 3-D vessel model is created by projecting the segmented 2-D vessel locations to the layer of the three middle voxels. A marching cube algorithm is applied to the initial model to construct a triangular mesh. The magnitude of the Gaussian derivative of the volumetric SD-OCT is combined with that of the vessel-aimed projection image to create the cost image. The globally optimal surfaces of the $3-\mathrm{D}$ vessels are achieved by solving a maximum flow problem on the constructed triangular-mesh-based graph from the combined cost image. An example of the $3-\mathrm{D}$ vessel segmentation result is shown in Fig. 2 p. 


\section{Experimental Methods and Results}

15 ONH-centered SD-OCT volumes from 15 subjects with glaucoma are acquired using a Cirrus ${ }^{T M}$ HD-OCT (Carl Zeiss Meditec) device. Each volume has $200 \times 200 \times 1024$ voxels corresponding to $6 \times 6 \times 2 \mathrm{~mm}^{3}$. Of the 15 volumes, 5 of them are randomly selected as the training set and 10 of them the test set. Each pixel in the vessel-aimed projection image is manually labeled as "vessel" or "non-vessel" with the help of experts. The small vessels by observation are excluded from the "vessel" category and labeled as "non-vessel".

The performance of the 2-D vessel segmentation is evaluated using Receiver Operating Characteristic (ROC) curves. Our present approach of the 2-D vessel segmentation is compared with Niemeijer's approach 2] in terms of the areas under the curves (AUC) for the regions around the NCO $( \pm 15$ pixels from the NCO boundary) and outside the NCO boundary. An example visual comparison of the 2-D and 3-D vessel segmentation between Niemeijer's and our approach is illustrated in the bottom row of Fig. 2, Fig. 2 2 and 2 r show the results of the 2-D and 3-D segmentation of our approach and Fig. 2f and 2h of Niemeijer's approach, respectively. As can be seen, the clear false positive near the NCO exists in the previous approach. However, in the present approach, it is greatly suppressed and the vessels around the NCO are detected. Table 1 demonstrates the 2-D quantitative segmentation results by comparing the AUCs of the region outside the $\mathrm{NCO}$ and the region around the $\mathrm{NCO}( \pm 15$ pixels from the $\mathrm{NCO}$ boundary) of the two algorithms. Our present algorithm gives a greater AUC in both regions, especially in the region around the NCO.

Table 1. AUC comparison of 2-D vessel segmentation of our and Niemeijer's algor.

\begin{tabular}{ccccc}
\hline \multicolumn{2}{c}{ AUC of the region around NCO } & & \multicolumn{2}{c}{ AUC of the region outside NCO } \\
\cline { 1 - 1 } \cline { 5 - 6 } Our algor. & Niemeijer's algor. & & Our algor. & Niemeijer's algor. \\
\hline 0.81 & 0.72 & & 0.84 & 0.81 \\
\hline
\end{tabular}

\section{Discussion and Conclusion}

We developed an approach for automatically segmenting the retinal blood vessels by focusing on the region around the $\mathrm{NCO}$ in ONH-centered SD-OCT volumes. To our knowledge, this is the first such algorithm to segment the blood vessels by utilizing a-priori NCO segmentation information, along with rotated Gabor wavelets and corresponding rotated NCO-based templates to suppress the false positive tendency near the NCO. The result is promising considering the difficulty of the vessel segmentation in ONH-centered SD-OCT volumes. Compared with the closest previous work, the $2-\mathrm{D}$ vessel segmentation results are greatly improved both visually and quantitatively, especially for the region around the NCO. However, the accuracy of the 2-D vessel segmentation is still not perfect. 
Some of the potential error sources include the following. 1) The OCT data were from patients with glaucoma and the image quality (noisiness and contrast) was not as good as that from normal scans, thereby causing the broken appearance of some of the smaller vessels. 2) The expert may not have always traced very small vessels they deemed "incomplete," whereas the algorithm would have found portions of these vessels, thus (incorrectly) causing the identification of these regions as false positives. In the future, for the 2 -D vessel pixel classification, the texture information around the NCO might be helpful for more accurately identifying the NCO and blood vessels. For the graph construction, using an arc-weighted graph [8] and considering the graph column crossing problem of vessel bifurcations 9] would also help improve the algorithm performance.

\section{References}

1. Wehbe, H., Ruggeri, M., Jiao, S., Gregori, G., Puliafito, C.A., Zhao, W.: Automatic retinal blood flow calculation using spectral domain optical coherence tomography. Optics Express 15(23), 15193-15206 (2007)

2. Niemeijer, M., Garvin, M.K., van Ginneken, B., Sonka, M., Abràmoff, M.D.: Vessel segmentation in 3D spectral OCT scans of the retina. In: Proc. of SPIE Medical Imaging: Image Processing, vol. 6914, p. 69141R (2008)

3. Hu, Z., Abràmoff, M.D., Kwon, Y.H., Lee, K., Garvin, M.K.: Automated segmentation of neural canal opening and optic cup in 3-D spectral optical coherence tomography volumes of the optic nerve head. Invest. Ophthalmol. Vis. Sci. (2010) (in press)

4. Garvin, M.K., Abràmoff, M.D., Wu, X., Russell, S.R., Burns, T.L., Sonka, M.: Automated 3-D intraretinal layer segmentation of macular spectral-domain optical coherence tomography images. IEEE Trans. Med. Imag. 28(9), 1436-1447 (2009)

5. Li, K., Wu, X., Chen, D.Z., Sonka, M.: Optimal surface segmentation in volumetric images - a graph-theoretic approach. IEEE Trans. Pattern Anal. Machine Intell. 28(1), 119-134 (2006)

6. Lee, K., Abràmoff, M.D., Niemeijer, M., Garvin, M.K., Sonka, M.: 3-D segmentation of retinal blood vessels in spectral-domain OCT volumes of the optic nerve head. In: Proc. of SPIE Medical Imaging: Biomedical Applications in Molecular, Structural, and Functional Imaging, vol. 7626, p. 76260V (2010)

7. Liu, C., Wechsler, H.: Gabor feature based classification using the enhanced fisher linear discriminant model for face recognition. IEEE Trans. Pattern Anal. Machine Intell. 11(4), 467-476 (2002)

8. Song, Q., Wu, X., Liu, Y., Smith, M., Buatti, J., Sonka, M.: Optimal graph search segmentation using arc-weighted graph for simultaneous surface detection of bladder and prostate. In: Yang, G.-Z., Hawkes, D., Rueckert, D., Noble, A., Taylor, C. (eds.) MICCAI 2009. LNCS, vol. 5762, pp. 827-835. Springer, Heidelberg (2009)

9. Yin, Y., Sonka, M.: Electric field theory based approach to search-direction line definition in image segmentation: Application to optimal femur-tibia cartilage segmentation in knee-joint 3-D MR. In: Proc. of SPIE Medical Imaging: Image Processing, vol. 7623 , p. $76230 \mathrm{~W}(2010)$ 